\title{
Organ-specific autoantibodies in Chinese patients newly diagnosed with type 1 diabetes mellitus
}

\author{
Shan Pan ${ }^{1,2)}$, Ting $\mathrm{Wu}^{1), 2)}$, Xiajie Shi ${ }^{1,2)}$, Zhiguo Xie ${ }^{1), 2)}$, Gan Huang ${ }^{1), 2)}$ and Zhiguang Zhou ${ }^{1), 2)}$ \\ 1) Department of Metabolism \& Endocrinology, The Second Xiangya Hospital, Central South University, Changsha, China \\ ${ }^{2)}$ Key Laboratory of Diabetes Immunology, Ministry of Education; National Clinical Research Center for Metabolic Diseases, \\ Changsha, China
}

\begin{abstract}
This study aims to investigate the prevalence of islet autoantibodies and other organ-specific autoantibodies in type 1 diabetes mellitus (T1DM) patients and characterize their clinical features. Glutamic acid decarboxylase antibody (GADA), insulinoma antigen 2 antibody (IA-2A), zinc transporter 8 antibody (ZnT8A) and tetraspanin7 antibody (TSPAN7A) were assayed by radioligand or luciferase immunoprecipitation system assays in 205 newly diagnosed acute-onset T1DM patients and 170 healthy controls. Other organ-specific autoantibodies, including thyroid peroxidase antibody (TPOA), thyroglobulin antibody (TGA), tissue transglutaminase antibody (tTGA) and 21-hydroxylase antibody (21-OHA), were also measured. The prevalence of GADA, IA-2A, ZnT8A, TSPAN7A, TPOA, TGA and 21-OHA was higher in T1DM patients than in healthy controls. The combinational assay of various islet autoantibodies could increase the frequency of autoantibody positivity in T1DM to $85.4 \%$. GADA+ IA-2A+ T1DM patients preferentially had TPOA and TGA, while IA-2A+ patients often had tTGA. Patients positive for two or more islet autoantibodies often had TPOA and TGA. BMI of multiple islet autoantibodypositive patients was lower than that of patients with single or no islet autoantibodies, and there were no significant differences in C-peptide and glycated hemoglobin between patients positive for islet autoantibodies combined with other organ-specific antibodies and noncombined patients. Younger female patients who were islet autoantibody positive were more likely to have TPOA and TGA. The frequency of Graves' disease was much higher in T1DM patients than in healthy controls. T1DM usually occurs together with other organ-specific autoantibodies. Measuring of other organ-specific autoantibodies will be beneficial for T1DM patients.
\end{abstract}

Key words: Type 1 diabetes mellitus, Islet autoantibody, Organ-specific autoantibody

T1DM is an autoimmune disease in which insulinproducing pancreatic islet $\beta$ cells are destroyed in genetically susceptible individuals, resulting in impaired blood glucose control and numerous pathological sequelae caused by chronic hyperglycemia [1]. Based on the most recent epidemiological survey, the "estimate of incidence of T1DM in all age groups in China during 2010-13", the estimated incidence of T1DM per 100,000 person years for all ages in China was 1.01 [2]. Several distinct islet autoantibodies may be detected in the serum of T1DM patients, including glutamic acid decarboxylase antibody (GADA), protein tyrosine phosphatase 2 autoantibody (IA-2A), zinc transporter- 8 autoantibody (ZnT8A) [3], and the recently reported tetraspanin7 antibody (TSPAN7A) [4]. Detection of these autoantibodies

Submitted Jan. 7, 2020; Accepted Mar. 9, 2020 as EJ20-0002 Released online in J-STAGE as advance publication Apr. 15, 2020 Correspondence to: Gan Huang, Department of Metabolism \& Endocrinology, The Second Xiangya Hospital, Central South University, No. 139 Renmin Road, Changsha, Hunan 410011, China. E-mail: huanggan007@csu.edu.cn is important for T1DM diagnosis and prediction.

T1DM patients are also at higher risk of other comorbid autoimmune diseases, including autoimmune thyroid disease (AITD), celiac disease (CD), and primary chronic adrenocortical hypofunction (e.g., Addison's disease, AD). Further, TIDM, AITD, and AD can also present as a triad constituting the main components of autoimmune polyendocrinopathy syndrome-II (APS-II) [5]. Autoimmune thyroid diseases, of which Hashimoto's thyroiditis and Graves' disease are the most common, is the most frequent autoimmune comorbidities in T1DM. The thyroid-specific immune damage of AITD is strongly associated with elevated serum thyroid peroxidase antibody (TPOA) [6]. In addition, thyroglobulin antibody (TGA) is also used as a reference index for the diagnosis of AITD. Celiac disease afflicts approximately $1 \%$ of the global population, but the overall incidence is low in China $(0.06 \%)$; however, the incidence in the northern provinces of Shandong, where wheat is the main staple food, reaches $0.76 \%$ [7]. Type 1 diabetes mellitus is considered a strong risk factor for $\mathrm{CD}$. 
Indeed, $\mathrm{CD}$ has been found in $0.97 \%$ to $16.7 \%$ of T1DM patients [8], a markedly higher rate than in nondiabetic patients. Tissue transglutaminase antibody (tTGA) is a specific antibody of intestinal epithelial cells and a serological marker of CD. Primary chronic adrenocortical dysfunction (e.g., $\mathrm{AD})$ is an autoimmune disorder mediated by cellular immunity that leads to destruction of the adrenal cortex, resulting in an inability to secrete sufficient quantities of cortisol. 21-Hydroxylase $(21-\mathrm{OH})$ is the main autoantigen implicated in $\mathrm{AD}$, and elevated serum $21-\mathrm{OH}$ antibody (21-OHA) is found in about $70 \%$ of patients [9].

Autoimmune comorbidities can exacerbate T1DM symptoms and introduce additional complications. For instance, subclinical hypothyroidism or hyperthyroidism due to AITD will increase the probability of hypoglycemia in T1DM, making blood glucose control more difficult $[10,11]$. Similarly, the digestive tract symptoms of $\mathrm{CD}$ can cause malnutrition, which will further disrupt blood sugar stability as well as stunt the growth and development of younger T1DM patients [12]. Addison's disease comorbid with T1DM can also impair efficient blood sugar control.

Several studies have confirmed that the prevalence of islet autoantibodies is related to race and genetic background $[13,14]$, but whether there are also genetic or ethnic differences in other organ-specific autoantibodies accompanying T1DM is unclear. We have previously studied autoimmune diabetes mellitus in Chinese adults [15], but there have been no reports on the autoantibody profile of classic T1DM in China. Therefore, we examined the profile of islet autoantibodies (GADA, IA-2A, ZnT8A, and TSPAN7A) as well as other organ-specific antibodies, such as TPOA, TGA, 21-OHA, and tTGA, in Chinese T1DM patients and matched controls. In addition, thyroid function, thyrotropin receptor antibody (TRAb), adrenocorticotropin (ACTH), and cortisol were measured in patients with abnormal antibody profiles to examine the correlations with patient clinical features.

\section{Methods}

\section{Study subjects}

The newly diagnosed acute-onset T1DM group consisted of 205 patients [119 males and 86 females; median age, 26 years (range, 1-67 years); median disease course, 2.0 months (range, 0-12 months)] receiving outpatient or inpatient treatment at the Second Xiangya Hospital of Central South University from January 2016 to December 2018. All met the 1999 diagnostic criteria and classification of the World Health Organization (WHO) for diabetes mellitus (initially presenting with ketosis or ketoacidosis and dependent on insulin for blood glucose control). The disease course refers to the time interval between the initial diagnosis of T1DM and the collection of blood samples for testing upon enrollment in the study. A healthy control group of 170 volunteers [92 males and 78 females; median age, 26.1 years (range, 15-38 years)] with no family history of diabetes was recruited from the Hunan Vocational College of Technology and the physical examination center of our hospital. All had normal fasting and $2 \mathrm{~h}$ postprandial blood glucose levels. There were no significant differences in age or sex constituent ratio between the healthy control group and T1DM group ( $p>0.05)$, indicating good comparability.

All participants provided written informed consent and the study protocol was approved by the Ethics Committee of the Second Xiangya Hospital of Central South University [(2014) under Ethics Approval No. (32)].

\section{Study methods \\ Acquisition of clinical data and collection of serum samples}

General information, including present and past illnesses as well as treatments, was collected from patient registration forms and by reviewing in-patient medical records. At least two study members completed and checked the registration forms.

Venous blood samples were collected in the morning after participants had fasted for at least $8 \mathrm{~h}$. The serum was separated and sent to the laboratory department of our hospital for testing of total cholesterol (TC), low density lipoprotein cholesterol (LDL-C), high density lipoprotein cholesterol (HDL-C), and triglyceride (TG) using an automatic biochemical analyzer (Type 7170, Hitachi, Tokyo, Japan). All participants submitted to a standard oral glucose tolerance test (OGTT, $75 \mathrm{~g}$ for adults and $1.75 \mathrm{~g} / \mathrm{kg}$ body weight up to $75 \mathrm{~g}$ for children). Serum samples were collected after $2 \mathrm{~h}$ as described. CPeptide (CP) was measured using a Bayer Centaur Immunoassay System automatic chemiluminescence analyzer and supporting reagents (Bayer, Leverkusen, Germany). The inter-assay and intra-assay variation coefficients (CVs) of the CP test were 3.7-4.1\% and $1.0-3.3 \%$, respectively. HbA1c was measured by automated liquid chromatography (Bio-Rad VARIANT-II Hemoglobin Testing System, United States). Serum free T3 (FT3), serum free T4 (FT4), thyroid stimulating hormone (TSH), TPOA, TGA, TRAb, and cortisol were tested with a Siemens XP automatic chemiluminescence analyzer and supporting reagents (Siemens, Munich, Germany). ACTH was tested with a Siemens 2000 automatic chemiluminescence analyzer and supporting reagents. The serum was stored at $-80^{\circ} \mathrm{C}$ for subsequent measurements of GADA, IA-2A, ZnT8A, TSPAN7A and other organ-specific antibodies. 


\section{Testing of serum GADA, IA-2A, and ZnT8A}

Recombinant human islet autoantigens were ${ }^{35} \mathrm{~S}$ methionine-labeled for the standardized radioligand assay in duplicate as previously described [16]. Briefly, islet autoantigens were transcribed/translated in vitro using the SP6 vector (or T7 for ZnT8) and rabbit reticulocyte lysate system. Labeled autoantigens were purified by gel filtration chromatography and coincubated with serum. Antigen-antibody complexes were precipitated by protein A agarose, subjected to high-flux washing, and counted using a liquid scintillation luminescence counter (MicroBeta Trilux, Wallac, Finland). The cutoff values of positivity for GADA, IA-2A, and ZnT8A were 18.0 U/mL, 3.3 U/mL World Health Organization units, and an antibody index of 0.011 , according to the 99th percentile observed in 405 healthy controls. Blood was drawn from the patients with positive antibodies again within a week to confirm their positivity and avoid false positivity. The sensitivity and specificity in our laboratory were $82 \%$ and $97.8 \%$ for GADA, $76 \%$ and $100 \%$ for IA-2A, and $72 \%$ and $100 \%$ specificity for ZnT8A, respectively, according to our results in the Islet Autoantibody Standardization Program (IASP) 2016. The intra-assay and inter-assay CVs were in the ranges of $4.3 \sim 10.2 \%$ and $8.2 \sim 11.9 \%$ for GADA, 3.2\% 9.7\% and 5.6\% 11.7\% for IA-2A, and $3.9 \% \sim 9.8 \%$ and $4.3 \% \sim 13.8 \%$ for $\mathrm{ZnT} 8 \mathrm{~A}$, respectively.

\section{TSPAN7A testing}

A previously established luciferase immunoprecipitation system assay (LIPS) was used for TSPAN7A testing [4]. Briefly, the TSPAN7-luciferase fusion protein was expressed in $293 \mathrm{~T}$ cells. Cells were homogenized in Glolysis buffer, and the lysate was coincubated with serum. Antigen-antibody complexes were precipitated with protein A-agarose and washed. Chromogenic substrate was then added, and the luciferase fluorescence was read by a MicroBeta LumiJET (PerkinElmer, Waltham, MA, USA). The results are expressed as the antibody index. The cutoff value was defined as the 99th percentile of the antibody index for 199 healthy controls $(\geq 0.024)$. The serum for testing was also subjected to double-tube testing, and the positive serum was then subjected to a competitive inhibition test. The intra-assay and inter-assay CVs were in the ranges of $6.3-12.4 \%$ and $14.9-35.7 \%$ for TSPAN7A, respectively.

\section{1-OHA and tTGA testing}

The 21-OH plasmid was kindly donated by Professor Kristina Åkesson, Department of Clinical Sciences, Lund University, Sweden, while the tTG plasmid was a generous donation from Professor Liping Yu, Barbara Davis Center for Childhood Diabetes, University of Colorado School of Medicine, United States. The testing method is described in a previous report [17], while the in vitro transcription/translation labeling and purification steps were similar to those for GADA, IA-2A, and ZnT8A testing. Briefly, a mixture of $5 \mu \mathrm{L}$ serum and $25 \mu \mathrm{L}$ diluted ${ }^{35} \mathrm{~S}-21-\mathrm{OH}$ (or ${ }^{35} \mathrm{~S}-\mathrm{tTGA}$ ) antigen (with a radioactivity count of $3.0 \times 10^{4} \mathrm{CPM}$ ) was incubated in 96-well micro-plates under horizontal oscillation for $24 \mathrm{~h}$ at $4{ }^{\circ} \mathrm{C}$, and then transferred to Millipore filtration plates precoated with protein A and sealed with fetal bovine serum. The Millipore filter plates were washed with TBST at high flux, and counted using a liquid scintillation luminescence counter. The results are expressed as the antibody index. The cutoff value was defined as the $99^{\text {th }}$ percentile of 179 healthy controls $(21-\mathrm{OHA} \geq 0.10$ and tTGA $\geq 0.02$ ). For quality control, each sample was subjected to a double-tube test. In cases with double-tube radioactivity count $\mathrm{CVs} \geq 30 \%$, the sample was retested. Sample batches with signal-to-noise $(\mathrm{S} / \mathrm{N})<15$ were also retested.

\section{Statistical analyses}

All statistical analyses were conducted using SPSS 20.0 and Microsoft Excel 2007. Measurement data are expressed as the mean \pm standard deviation or median [range] and enumeration data are expressed as the number of positive cases and constituent ratio/rate. Ratios/ numbers were compared by the chi-square test or Fisher's exact probability test if the expected number was $<5$. Normally distributed continuous variables were compared by one-way ANOVA, and nonnormally distributed continuous variables were compared using a nonparametric test. A $p<0.05$ (two-tailed) was considered significant for all tests.

\section{Results}

\section{Positivity of islet autoantibodies and other organ- specific autoantibodies in T1DM patients}

Of the 205 newly diagnosed T1DM patients enrolled, $144(70.2 \%)$ were positive for GADA, 88 (42.9\%) for IA-2A, $63(30.7 \%)$ for ZnT8A, and 60 (29.3\%) for TSPAN7A (Fig. 1). These positivity rates were significantly higher than those in the healthy control group [1 of $170(0.6 \%), 0,0$, and 2 of $170(1.2 \%)$, respectively, $p=0.0000]$. Similarly, positivity rates were significantly higher in the T1DM group than in the healthy control group for the organ-specific autoantibodies TPOA [51/205 (24.9\%) vs. $13 / 170(7.6 \%) ; \mathrm{x}^{2}=19.49, p=$ $0.0000]$, TGA [38/205 (18.5\%) vs. 10/170 (5.9\%); $\mathrm{x}^{2}=$ 13.33, $p=0.0003]$, and 21-OHA [6/205 (2.9\%) vs. 0/170 $\left.(0) ; x^{2}=4.19, p=0.0405\right]$. Positivity for tTGA was also numerically higher in the T1DM group than in the healthy control group, but the difference did not reach statistical significance $[7 / 205(3.4 \%)$ vs. $1 / 170(0.6 \%)$; $\left.\mathrm{x}^{2}=2.33, p=0.1268\right]$. According to onset age, we 
further divided the T1DM patients into two subgroups as follows: the juvenile group ( $\leq 20$ years) and the adult group ( $>20$ years). The prevalence of IA-2A and ZnT8A in the juvenile group was higher than that in the adult group $(p<0.001)$. See Table 1 .

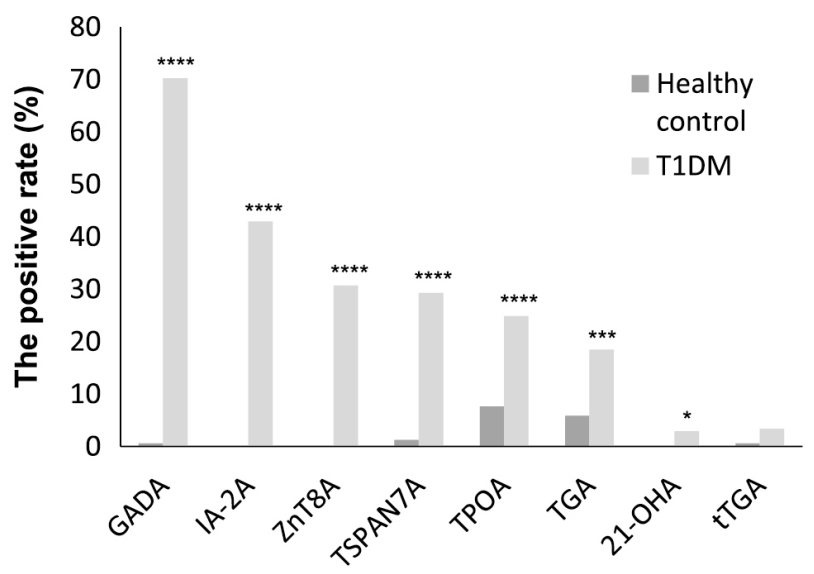

Fig. 1 Positive rate of islet autoantibodies and other organspecific antibodies in patients with T1DM and healthy controls. Compared with healthy control, $* p<0.05,{ }^{* * *} p$ $<0.001, * * * * p<0.0001$.

\section{Combined detection of islet autoantibodies}

In separate tests for the presence of multiple islet antibodies (Table 2), the GADA-positive rate was $70.2 \%$, the GADA or IA-2A rate was $81.0 \%$, and the GADA, IA-2A, or ZnT8A rate was $82.9 \%$. The combinational assay of various islet autoantibodies could increase the frequency of autoantibody positivity in T1DM to $85.4 \%$. However, the GADA, IA-2A, ZnT8A or TSPAN7A rate $(85.4 \%)$ did not differ from the GADA, IA-2A or ZnT8A rate $(82.9 \%, p>0.05)$. Adding TSPAN7A did not increase the positivity rate if GADA, IA-2A or ZnT8A have been tested.

\section{Positive overlap among islet autoantibodies and other organ-specific autoantibodies}

As shown in Table 3, T1DM patients positive for GADA were also more likely to show positivity for TPOA or TGA than GADA-negative patients ( $p=0.004$ and 0.037 , respectively). Patients positive for IA-2A were more likely to be positive for TPOA $(p=0.020)$, TGA $(p=0.015)$ or tTGA $(p=0.002)$ than IA-2Anegative patients. In addition, 7 tTGA-positive patients were also positive for IA-2A, 6 patients were positive for

Table 1 Comparison of antibody positive rates between the juvenile group and the adult group

\begin{tabular}{|c|c|c|c|c|c|}
\hline \multirow{2}{*}{ Antibody combination } & \multicolumn{2}{|c|}{ the juvenile group $(n=81)$} & \multicolumn{2}{|c|}{ the adult group $(n=124)$} & \multirow{2}{*}{$p$ value } \\
\hline & Number of positive cases & Positive rate $(\%)$ & Number of positive cases & Positive rate $(\%)$ & \\
\hline GADA & 59 & 72.8 & 85 & 68.5 & 0.536 \\
\hline IA-2A & 51 & 63.0 & 37 & 29.8 & 0.000 \\
\hline $\mathrm{ZnT} 8 \mathrm{~A}$ & 41 & 50.6 & 22 & 17.7 & 0.000 \\
\hline TSPAN7A & 26 & 32.1 & 34 & 27.4 & 0.531 \\
\hline TPOA & 18 & 22.2 & 33 & 26.6 & 0.512 \\
\hline TGA & 17 & 21.0 & 21 & 16.9 & 0.469 \\
\hline 21-OHA & 3 & 3.7 & 3 & 2.4 & 0.683 \\
\hline tTGA & 4 & 4.9 & 3 & 2.4 & 0.277 \\
\hline
\end{tabular}

Table 2 Positive rate of combined detection of islet autoantibody in patients with T1DM

\begin{tabular}{lcc}
\hline Antibody combination & Number of positive cases & Positive rate $(\%)$ \\
\hline GADA & 144 & 70.2 \\
IA-2A & 88 & 42.9 \\
ZnT8A & 63 & 30.7 \\
TSPAN7A & 60 & 29.3 \\
GADA or ZnT8A & 157 & 76.6 \\
GADA or IA-2A & 166 & $81.0^{*}$ \\
\hline GADA, IA-2A or ZnT8A & 170 & $82.9 * *$ \\
\hline GADA, IA-2A, ZnT8A or TSPAN7A & 175 & $85.4 * *$ \\
\hline
\end{tabular}

Note: compared with the positive rate of separated test for GADA, $* p<0.05, * * p<0.01$. 
GADA, and 3 patients were positive for ZnT8A. Further grouping analysis showed that TPOA- and TGA-positive rates were higher in patients double- or triple-positive for islet autoantibodies compared to those showing positivity for only one islet autoantibody $(p<0.05 \sim 0.01)$. See Table 4.

\section{Associations with clinical characteristics}

As shown in Table 5, the T1DM patients were divided into negative, single GADA-positive, single IA-2Apositive, single ZnT8A-positive and single TSPAN7Apositive subgroups by the types of islet autoantibody. Differences in the following clinical characteristics were examined: age, sex ratio, body mass index (BMI), disease course, waist-hip ratio, systolic blood pressure (SBP), diastolic blood pressure (DBP), glycosylated hemoglobin (HbA1c), fasting $\mathrm{CP}$, postprandial $\mathrm{CP}$, and blood lipids. The single GADA-positive subgroup showed lower post- prandial CP than the negative subgroup, and there were no significant differences in clinical characteristics among the single IA-2A-, ZnT8A-, and TSPAN7Apositive subgroups and the negative subgroup.

According to the number of islet autoantibodies presented, the 205 patients were divided into negative, single-positive, double-positive, triple-positive, and quadruple-positive subgroups. Both double- and triplepositive subgroups exhibited a lower BMI than the negative subgroup $(p<0.05)$, while the waist-to-hip ratio was lower in the single-, double-, and triple-positive subgroups than in the negative subgroup $(p<0.05)$. There were no significant differences in clinical characteristics between double- and triple-positive subgroups $(p>$ 0.05). See Table 6.

According to the positivity status of islet autoantibodies and other organ-specific autoantibodies, the T1DM patients were divided into 6 subgroups: IA-OS- sub-

Table 3 T1DM patients with different islet autoantibodies positive combined with other organ-specific autoantibodies [percentage (number)]

\begin{tabular}{|c|c|c|c|c|}
\hline & $\mathrm{TPOA}+(n=51)$ & $\mathrm{TGA}+(n=38)$ & $21-\mathrm{OHA}+(n=6)$ & $\mathrm{tTGA}+(n=7)$ \\
\hline $\mathrm{GADA}+(n=144)$ & $30.6 \%(44)$ & $22.9 \%(33)$ & $2.8 \%(4)$ & $4.2 \%(6)$ \\
\hline GADA- $(n=61)$ & $11.5 \%(7)$ & $8.2 \%(5)$ & $3.3 \%(2)$ & $1.6 \%(1)$ \\
\hline$x^{2}$ value & 8.347 & 4.337 & - & - \\
\hline$p$ value & 0.004 & 0.037 & 1.000 & 0.677 \\
\hline $\mathrm{IA}-2 \mathrm{~A}+(n=88)$ & $33 \%(29)$ & $26.1 \%(23)$ & $4.5 \%(4)$ & $8.0 \%(7)$ \\
\hline IA-2A- $(n=117)$ & $18.8 \%(22)$ & $12.8 \%(15)$ & $1.7 \%(2)$ & $0 \%(0)$ \\
\hline$x^{2}$ value & 5.382 & 5.897 & - & - \\
\hline$p$ value & 0.020 & 0.015 & 0.405 & 0.002 \\
\hline $\mathrm{ZnT} 8 \mathrm{~A}+(n=63)$ & $31.7 \%(20)$ & $25.4 \%(16)$ & $3.2 \%(2)$ & $4.8 \%(3)$ \\
\hline ZnT8A- $(n=142)$ & $22.5 \%(31)$ & $15.5 \%(22)$ & $2.8 \%(4)$ & $2.8 \%(4)$ \\
\hline$x^{2}$ value & 1.956 & 2.835 & - & - \\
\hline$p$ value & 0.162 & 0.092 & 1.000 & 0.679 \\
\hline TSPAN7A $+(n=60)$ & $26.7 \%(16)$ & $21.7 \%(13)$ & $5.0 \%(3)$ & $5.0 \%(3)$ \\
\hline TSPAN7A- $(n=145)$ & $24.1 \%(35)$ & $17.2 \%(25)$ & $2.1 \%(3)$ & $2.1 \%(3)$ \\
\hline$x^{2}$ value & 0.145 & 0.550 & - & - \\
\hline$p$ value & 0.724 & 0.554 & 0.361 & 0.361 \\
\hline
\end{tabular}

Table 4 T1DM patients with different amounts of islet autoantibodies positive combined with other organ-specific autoantibodies

\begin{tabular}{lllrc}
\hline & TPOA & TGA & 21-OHA & tTGA \\
\hline Single-positive $(n=81)$ & $14.8 \%(12)$ & $9.8 \%(8)$ & $1.2 \%(1)$ & $1.2 \%(1)$ \\
Double-positive $(n=53)$ & $41.5 \%(22)^{*}$ & $28.3 \%(15)^{*}$ & $5.7 \%(3)$ & $5.7 \%(3)$ \\
Triple-positive $(n=36)$ & $33.3 \%(12)^{* *}$ & $30.5 \%(11)^{*}$ & $0 \%(0)$ & $0 \%(0)$ \\
Quadruple-positive $(n=21)$ & $23.8 \%(5)$ & $28.6 \%(6)$ & $0 \%(0)$ & $0 \%(0)$ \\
\hline
\end{tabular}

Note: compared with the single-positive antibody group, $* p<0.05, * * p<0.01$. 
Table 5 Clinical characteristics of T1DM patients with single islet autoantibody positive

\begin{tabular}{|c|c|c|c|c|c|}
\hline & $\begin{array}{c}\text { Negative antibody } \\
\text { patients }\end{array}$ & GADA+ & IA-2A+ & ZnT8A+ & TSPAN7A+ \\
\hline$N$ & 35 & 55 & 9 & 4 & 5 \\
\hline Sex (male/female) & $25 / 10$ & $38 / 17$ & $5 / 4$ & $2 / 2$ & $3 / 2$ \\
\hline Age (years) & $21.44 \pm 10.25$ & $29.11 \pm 14.24$ & $23.89 \pm 20.07$ & $22.50 \pm 7.77$ & $37.80 \pm 20.50$ \\
\hline disease course (month) & $4.0(0.5-10)$ & $2.0(0.6-7.0)$ & $4.0(1.0-9.0)$ & $1.0(0.33-1.75)$ & $3.0(1.0-10.5)$ \\
\hline BMI $\left(\mathrm{kg} / \mathrm{m}^{2}\right)$ & $21.44 \pm 10.25$ & $19.56 \pm 3.04$ & $17.27 \pm 3.01$ & $21.82 \pm 4.41$ & $19.01 \pm 2.41$ \\
\hline waist-hip Ratio & $0.858 \pm 0.053$ & $0.724 \pm 0.311$ & $0.658 \pm 0.377$ & $0.883 \pm 0.053$ & $0.850 \pm 0.007$ \\
\hline SBP (mmHg) & $113.3 \pm 15.8$ & $110.5 \pm 13.7$ & $108.0 \pm 12.5 .8$ & $110.2 \pm 13.6$ & $111.8 \pm 19.7$ \\
\hline $\mathrm{DBP}(\mathrm{mmHg})$ & $72.1 \pm 10.3$ & $72.7 \pm 10.8$ & $69.6 \pm 11.7$ & $73.7 \pm 5.3$ & $72.4 \pm 11.5$ \\
\hline HbAlc (\%) & $9.72 \pm 3.39$ & $10.13 \pm 2.41$ & $10.40 \pm 3.25$ & $10.23 \pm 2.19$ & $10.50 \pm 2.52$ \\
\hline fasting $\mathrm{CP}(\mathrm{pmol} / \mathrm{L})$ & $84(5.5-128.9)$ & $112.6(39.8-139.2)$ & $78.4(10.1-118.2)$ & $105.4(54.4-237.1)$ & $168.5(9.2-168.5)$ \\
\hline $\begin{array}{l}\text { postprandial CP } \\
(\mathrm{pmol} / \mathrm{L})\end{array}$ & $168.3(6.1-277.2)$ & $133.0(77.0-256.8)^{*}$ & $118.7(9.9-320.1)$ & $316.0(83.3-374.0)$ & $314.9(8.6-314.9)$ \\
\hline $\mathrm{TG}(\mathrm{mmol} / \mathrm{L})$ & $1.19 \pm 0.76$ & $1.08 \pm 0.84$ & $0.85 \pm 0.35$ & $1.63 \pm 1.87$ & $1.26 \pm 0.50$ \\
\hline $\mathrm{TC}(\mathrm{mmol} / \mathrm{L})$ & $4.02 \pm 1.01$ & $4.13 \pm 1.00$ & $4.51 \pm 0.56$ & $3.38 \pm 0.96$ & $4.73 \pm 0.72$ \\
\hline HDL-C (mmol/L) & $1.19 \pm 0.31$ & $1.27 \pm 0.37$ & $1.41 \pm 0.45$ & $1.11 \pm 0.73$ & $1.41 \pm 0.17$ \\
\hline LDL-C (mmol/L) & $2.48 \pm 0.91$ & $2.46 \pm 0.90$ & $2.65 \pm 0.66$ & $1.76 \pm 0.74$ & $2.88 \pm 0.63$ \\
\hline
\end{tabular}

Note: compared with the negative antibody patients, ${ }^{*} p<0.05$.

group (both islet autoantibodies and other organ-specific autoantibodies are negative); IA+OS - subgroup (any islet autoantibody is positive and other organ-specific autoantibodies are negative); IA+TPO+ subgroup (any islet autoantibody is positive combined with TPOA positivity); IA+TGA + subgroup (any islet autoantibody is positive combined with TGA positivity); IA+21-OHA+ subgroup (any islet autoantibody is positive combined with 21-OHA positivity); and IA+tTGA+ subgroup (any islet autoantibody is positive combined with tTGA positivity). The data showed that compared with the IA-OSor IA+OS- subgroup, the IA+TPO + and IA+TGA+ subgroups had more female patients $(p<0.05)$. Patients in the $\mathrm{IA}+\mathrm{TGA}+$ and $\mathrm{IA}+\mathrm{tTGA}+$ subgroups were younger than those in the IA-OS- subgroup $(p<0.05)$. There were no significant differences in clinical characteristics among these 6 subgroups. See Table 7.

Among the 205 patients, 51 were positive for TPOA, 38 for TGA, 6 for 21-OHA, and 7 for tTGA, while 142 cases were negative for all four antibodies. There were no significant differences in clinical characteristics among these 5 subgroups $(p>0.05)$.

Serum FT3, FT4, TSH, and TRAb levels were measured in 58 T1DM patients positive for TPOA or TGA (tested value $>60 \mathrm{U} / \mathrm{mL}$ ), of whom 14 were confirmed to have hypothyroidism (including 10 patients with subclinical hypothyroidism) due to Hashimoto's thyroiditis, accounting for $6.8 \%(14 / 205)$ of the total patient group.
This group of TPOA- or TGA-positive patients also included 12 with hyperthyroidism due to Graves' disease (including 5 patients with subclinical hyperthyroidism), of whom 9 had abnormal TRAb (tested value $>1.5 \mathrm{IU} / \mathrm{L}$ ), accounting for $4.4 \%(9 / 205)$ of the total patient group. Among 15 healthy controls positive for TPOA or TGA, 5 had hypothyroidism (including 4 patients with subclinical hypothyroidism), accounting for $2.9 \%$ of the total control group. There were also 3 patients with hyperthyroidism (including 2 patients with subclinical hyperthyroidism), of whom 1 patient had abnormal TRAb (0.6\% of the total control population). There was a significant difference in Graves' disease frequency between the T1DM group and the healthy control group $(p=0.0252)$. Hashimoto's thyroiditis was also more frequent in the T1DM group than in the healthy control group, but the difference did not reach significance $(p=0.0874)$. Among the 6 T1DM patients positive for 21-OHA, one also had elevated serum cortisol $(893.6 \mathrm{nmol} / \mathrm{L} v s$. reference value 85.3-618.0 nmol/L), while ACTH values were all within the reference range.

\section{Discussion}

T1DM may be complicated by autoimmune diseases targeting other endocrine organs, such as AITD and primary chronic adrenocortical hypofunction (Addison's disease), as well as autoimmune diseases targeting non- 
Table 6 Clinical characteristics of T1DM patients with different amounts of islet autoantibodies

\begin{tabular}{|c|c|c|c|c|c|}
\hline & $\begin{array}{l}\text { Negative antibody } \\
\text { group }\end{array}$ & Single-positive group & $\begin{array}{l}\text { Double-positive } \\
\text { group }\end{array}$ & Triple-positive group & $\begin{array}{l}\text { Quadruple-positive } \\
\text { group }\end{array}$ \\
\hline$N$ & 35 & 81 & 53 & 36 & 21 \\
\hline $\begin{array}{l}\text { Sex (male/ } \\
\text { female) }\end{array}$ & $25 / 10$ & $55 / 26$ & $25 / 28^{\#}$ & $14 / 22 * * \#$ & $10 / 11$ \\
\hline Age (years) & $21.44 \pm 10.25$ & $19.45 \pm 3.24$ & $18.85 \pm 3.36$ & $18.10 \pm 3.57$ & $18.22 \pm 3.38$ \\
\hline $\begin{array}{l}\text { disease course } \\
\text { (month) }\end{array}$ & $4.0(0.5-10)$ & $2.0(1.0-7.5)$ & $2.0(0.6-5.0)$ & $2.5(1.0-5.8)$ & $2.0(1.0-5.8)$ \\
\hline BMI $\left(\mathrm{kg} / \mathrm{m}^{2}\right)$ & $21.44 \pm 10.25$ & $19.46 \pm 3.25$ & $18.85 \pm 3.36^{*}$ & $18.10 \pm 3.57 *$ & $18.13 \pm 3.16^{*}$ \\
\hline waist-hip Ratio & $0.858 \pm 0.053$ & $0.853 \pm 0.074^{*}$ & $0.847 \pm 0.072 *$ & $0.813 \pm 0.053^{*}$ & $0.815 \pm 0.052 *$ \\
\hline $\mathrm{SBP}(\mathrm{mmHg})$ & $113.31 \pm 15.83$ & $110.12 \pm 13.35$ & $113.45 \pm 14.80$ & $106.86 \pm 12.78$ & $107.01 \pm 13.08$ \\
\hline DBP (mmHg) & $72.14 \pm 10.32$ & $72.04 \pm 10.49$ & $71.10 \pm 9.85$ & $69.57 \pm 11.15$ & $69.88 \pm 11.64$ \\
\hline HbA1c (\%) & $9.72 \pm 3.39$ & $10.10 \pm 2.56$ & $10.26 \pm 3.16$ & $10.16 \pm 3.10$ & $10.27 \pm 3.12$ \\
\hline $\begin{array}{l}\text { fasting CP } \\
(\mathrm{pmol} / \mathrm{L})\end{array}$ & $84.0(5.5-128.9)$ & $90.2(27.9-148.5)$ & $81.7(9.2-141.6)$ & $61.4(22.4-112.7)$ & $71.4(30.3-112.7)$ \\
\hline $\begin{array}{l}\text { postprandial CP } \\
(\mathrm{pmol} / \mathrm{L})\end{array}$ & $168.3(6.1-277.2)$ & $173.6(61.1-286.1)$ & $118.75(10.0-320.1)$ & $123.3(0-214.7)$ & $120.8(0-223.3)$ \\
\hline $\mathrm{TG}(\mathrm{mmol} / \mathrm{L})$ & $1.19 \pm 0.76$ & $1.21 \pm 1.11$ & $1.05 \pm 0.81$ & $0.97 \pm 0.82$ & $0.98 \pm 0.81$ \\
\hline $\mathrm{TC}(\mathrm{mmol} / \mathrm{L})$ & $4.02 \pm 1.01$ & $4.07 \pm 0.93$ & $4.18 \pm 1.06$ & $4.21 \pm 1.34$ & $4.09 \pm 1.13$ \\
\hline $\begin{array}{l}\text { HDL-C } \\
(\mathrm{mmol} / \mathrm{L})\end{array}$ & $1.19 \pm 0.31$ & $1.25 \pm 0.42$ & $1.24 \pm 0.34$ & $1.36 \pm 0.56$ & $1.23 \pm 0.47$ \\
\hline LDL-C (mmol/L) & $2.48 \pm 0.91$ & $2.44 \pm 0.86$ & $2.47 \pm 0.92$ & $2.44 \pm 1.19$ & $2.41 \pm 1.23$ \\
\hline
\end{tabular}

Note: compared with the negative antibody group, ${ }^{*} p<0.05,{ }^{* *} p<0.01$; compared with the single-positive antibody group, ${ }^{\#} p<0.05$, \# $p<0.01$.

Table 7 Clinical characteristics of T1DM patients with different positive status of islet autoantibodies and other organ-specific autoantibodies

\begin{tabular}{|c|c|c|c|c|c|c|}
\hline & IA-OS- & $\mathrm{IA}+\mathrm{OS}-$ & $\mathrm{IA}+\mathrm{TPO}+$ & IA+TGA+ & $\mathrm{IA}+21-\mathrm{OHA}+$ & $\mathrm{IA}+\mathrm{tTGA}+$ \\
\hline$N$ & 24 & 118 & 46 & 34 & 5 & 7 \\
\hline Sex (male/female) & $18 / 6$ & $71 / 47$ & $19 / 27 * \#$ & $12 / 22 * \#$ & $3 / 2$ & $3 / 4$ \\
\hline Age (years) & $32.17 \pm 13.01$ & $26.17 \pm 16.66$ & $26.85 \pm 12.98$ & $22.44 \pm 12.63^{*}$ & $23.40 \pm 20.47$ & $17.28 \pm 13.58^{*}$ \\
\hline disease course (month) & $4.0(0.4-9)$ & $2.0(1.0-7.0)$ & $2.0(1.0-6.3)$ & $2.0(1.0-5.0)$ & $4.0(0.9-5.5)$ & $4.0(1.0-7.0)$ \\
\hline BMI $\left(\mathrm{kg} / \mathrm{m}^{2}\right)$ & $22.48 \pm 12.22$ & $18.76 \pm 3.23$ & $19.13 \pm 3.46$ & $19.41 \pm 4.11$ & $18.14 \pm 1.98$ & $18.15 \pm 3.52$ \\
\hline waist-hip Ratio & $0.779 \pm 0.245$ & $0.683 \pm 0.344$ & $0.830 \pm 0.061$ & $0.829 \pm 0.075$ & $0.836 \pm 0.068$ & $0.840 \pm 0.952$ \\
\hline SBP (mmHg) & $113.46 \pm 14.70$ & $109.08 \pm 12.88$ & $113.67 \pm 16.29$ & $115.29 \pm 15.91$ & $103.25 \pm 9.64$ & $108.67 \pm 12.58$ \\
\hline DBP (mmHg) & $72.08 \pm 9.90$ & $70.79 \pm 9.71$ & $72.63 \pm 12.23$ & $72.29 \pm 12.44$ & $68.75 \pm 5.86$ & $69.67 \pm 4.50$ \\
\hline HbAlc (\%) & $9.73 \pm 3.76$ & $10.21 \pm 2.91$ & $10.19 \pm 2.71$ & $10.34 \pm 3.08$ & $9.12 \pm 1.58$ & $8.25 \pm 1.68$ \\
\hline fasting $\mathrm{CP}(\mathrm{pmol} / \mathrm{L})$ & $\begin{array}{c}83.8 \\
(30.8-128.9)\end{array}$ & $\begin{array}{c}80.9 \\
(13.5-144.1)\end{array}$ & $\begin{array}{c}88.5 \\
(35.6-157.1)\end{array}$ & $\begin{array}{c}81.9 \\
(34.6-175.8)\end{array}$ & $\begin{array}{c}21.5 \\
(17.9-195.4)\end{array}$ & $\begin{array}{c}77.7 \\
(14.4-130.2)\end{array}$ \\
\hline postprandial CP (pmol/L) & $\begin{array}{c}149.7 \\
(66.3-277.7)\end{array}$ & $\begin{array}{c}120.8 \\
(5.5-260.8)\end{array}$ & $\begin{array}{c}178.2 \\
(90.2-224.7)\end{array}$ & $\begin{array}{c}179.8 \\
(85.4-293.8)\end{array}$ & $\begin{array}{c}5.0 \\
(5-596.9)\end{array}$ & $\begin{array}{c}107.5 \\
(5.0-279.8)\end{array}$ \\
\hline TG (mmol/L) & $1.11 \pm 0.87$ & $1.21 \pm 1.13$ & $0.94 \pm 0.51$ & $1.09 \pm 0.52$ & $1.52 \pm 0.49$ & $0.72 \pm 0.38$ \\
\hline $\mathrm{TC}(\mathrm{mmol} / \mathrm{L})$ & $3.90 \pm 0.86$ & $4.27 \pm 1.06$ & $3.91 \pm 1.09$ & $3.99 \pm 0.86$ & $2.91 \pm 1.00$ & $3.26 \pm 0.44$ \\
\hline HDL-C (mmol/L) & $1.16 \pm 0.26$ & $1.27 \pm 0.46$ & $1.26 \pm 0.36$ & $1.22 \pm 0.34$ & $1.32 \pm 0.37$ & $1.26 \pm 0.37$ \\
\hline LDL-C (mmol/L) & $2.32 \pm 0.86$ & $2.58 \pm 0.96$ & $2.20 \pm 0.91$ & $2.28 \pm 0.80$ & $1.19 \pm 0.85$ & $1.74 \pm 0.32$ \\
\hline
\end{tabular}

Note: compared with IA-OS-, * $p<0.05$; compared with IA+OS-, ${ }^{\#} p<0.05$. 
endocrine organs, such as systemic lupus erythematosus (SLE), vitiligo, and CD [18, 19]. Previous studies have found that $15-30 \%$ of T1DM patients have comorbid autoimmune thyroid diseases, $0.97-16.7 \%$, celiac disease; and $0.5-1 \%$, Addison's disease [20], as evidenced by the presence of autoantibodies such as TPOA, TGA, tTGA and 21-OHA. A substantial proportion of T1DM patients seropositive for other organ-specific autoantibodies will progress to a clinical condition more complex and intractable than T1DM alone, so it is critical to identify subclinical comorbidities as early as possible by autoantibody screening [21]. In addition, careful monitoring of patients positive for organ-specific autoantibodies will facilitate early diagnosis of related diseases and timely clinical intervention.

Consistent with previous studies [22], positivity rates for islet autoantibodies were substantially higher in T1DM patients than in controls. GADA is a commonly detected islet autoantibody in newly diagnosed T1DM, followed by IA-2A and ZnT8A, and $82.9 \%$ of patients were positive for at least one of the three. Therefore, combined testing will provide a more sensitive diagnosis of T1DM and may reveal the severity of the immune pathology. Tetraspanin-7 is a newly confirmed T1DM autoantigen [23]. The autoantibody TSPAN7A is more frequently detected together with GADA, IA-2A, or ZnT8A than alone [24]. In this study, among 35 T1DM patients negative for GADA, IA-2A, and ZnT8A, 5 screened positive for TSPAN7A, indicating that T1DM may be associated with TSPAN7A alone in a minority of Chinese patients. This TSPAN7A-positive rate was higher than that reported previously [22], likely because this study included only T1DM patients diagnosed within the past year and positivity rates decrease with disease duration. Analyzing the association between the onset age of T1DM and autoantibodies, the results showed that both IA-2A and ZnT8A tend to appear in younger T1DM patients, and these results are consistent with previous research [25].

Positivity rates for TPOA, TGA, and 21-OHA were significantly higher in patients than in healthy controls, and tTGA was also detected more frequently in patients (although the difference did not reach significance, possibly due to an inherently low positivity rate and a limited sample size). Collectively, these findings indicate that Chinese T1DM patients are also prone to other organ-specific autoimmune diseases, presumably related to the expansion of antibody recognition epitopes among different antigen molecules. Although previous studies have found that patients positive for IA-2A are at higher risk of thyroid dysfunction [26], there have been no reports that IA-2A-positive T1DM patients are more likely to be positive for thyroid antibodies. We found that patients positive for GADA or IA-2A were more likely to exhibit positivity for TPOA or TGA, suggesting that those patients are prone to develop comorbid thyroid autoimmune diseases. In a study of 2,433 newly diagnosed T1DM patients in Sweden, Jonsdottir et al. [27] found that ZnT8A positivity and GADA positivity were associated with TPOA and TGA detection. An association between ZnT8A and TPOA positivity was not observed in the current study, possibly due to the limited sample size or the difference in genetic background. Previous studies have confirmed that autoimmune diabetes mellitus patients with high GADA titers are more prone to comorbid thyroid autoimmunity $[28,29]$, and in the current study, T1DM patients with two or more islet autoantibodies were more likely to be positive for TPOA or TGA. In addition, the CD marker tTGA was found more frequently in IA-2A-positive T1DM patients, suggesting that such patients should be examined for CD by tTGA screening. While the reason for this association between IA-2A positivity and CD is unclear, it may suggest stronger and more extensive autoimmunity in IA-2A-positive T1DM patients. Finally, no correlation was found between 21-OHA and islet autoantibody positivity; presumably, the sample population was small. These findings may define a new antibody profile for diagnosis.

We also presented the associations with clinical characteristics. For islet autoantibodies, except for the level of postprandial $\mathrm{CP}$, there was no difference in clinical characteristics among patients with various types of single-antibody positivity. BMI was lower in T1DM patients with multiple islet autoantibodies, and T1DM patients with two or three islet autoantibodies were more often females than males; however, there were no significant differences in CP level, a key indicator of islet function, or glycosylated hemoglobin, a key indicator of blood glucose control among patients with different islet antibody numbers. The reason may be due to the limited sample size. Alternatively, the type or number of islet antibodies present may not be the decisive factor affecting islet function. On the other hand, younger female patients with islet autoantibody positivity were more likely to present TPOA or TGA, suggesting that those patients have a risk of comorbid thyroid autoimmune diseases. Moreover, patients with islet autoantibodies combined with tTGA positivity were relatively younger. The presence of other organ-specific autoantibodies did not significantly affect the clinical features of diabetes, which suggests the specificity of organ involvement in autoimmune diseases or that antibodies are an indicator of the humoral immune response in cell-mediated immune injury.

We further tested thyroid function and thyrotropin 
receptor antibody positivity in T1DM patients positive for TPOA or TGA and found that T1DM was complicated by Hashimoto's thyroiditis in $6.8 \%$ of cases and Graves' disease in $4.4 \%$, far higher than in the general population. Collectively, these results strongly suggest that Chinese T1DM patients are at markedly greater risk of autoimmune thyroid diseases.

Although 21-OHA positivity was also higher in patients than in healthy controls, no adrenocortical hypofunction was found in patients by subsequent $\mathrm{ACTH}$ and cortisol testings, possibly due to the small number of 21OHA-positive patients. Since this is a cross-sectional study, no further follow-up of adrenal gland function or $\mathrm{CD}$ was conducted. Therefore, a larger-scale multicenter prospective study with improved follow-up is warranted to assess the effects of these other diseases on T1DM.

\section{Funding information}

This study was supported by the National Key R\&D Program of China (2018YFC1315603, 2016YFE0107500), the National Natural Science Foundation of China (81820108007, 81600649) and Science and Technology Major Project of Hunan Province (2017SK1020).

\section{Acknowledgements}

We thank Prof. John C. Hutton for providing the recombinant ZnT8 plasmid, Prof. Bill Hagopian and for providing the recombinant hGAD65 and IA-2 plasmid, Prof. Liping Yu for providing the recombinant tTG plasmid and Prof. Åkesson for providing the recombinant 21-OH plasmid.

\section{Declaration of Competing Interest}

The authors declare that there are no conflicts of interest.

\section{Authors' Contribution Statement}

Blood sample collection, statistical analysis, paper writing were done by Dr. Shan Pan and Dr. Ting Wu. Prof. Gan Huang designed the study. Prof. Zhiguo Xie and Dr. Xiajie Shi all participated in revision. All contributing members were read, revised, and approved this article.

\section{Data Availability Statement}

The data that support the findings of this study are available from the corresponding author upon reasonable request.

\section{References}

1. Atkinson MA, Eisenbarth GS (2001) Type 1 diabetes: new perspectives on disease pathogenesis and treatment. Lancet 358: 221-229.

2. Weng J, Zhou Z, Guo L, Zhu D, Ji L, et al. (2018) Incidence of type 1 diabetes in China, 2010-13: population based study. BMJ 360: j5295.

3. Rogowicz-Frontczak A, Pilacinski S, Wyka K, WieruszWysocka B, Zozulinska-Ziolkiewicz D (2018) Zinc transporter 8 autoantibodies (ZnT8-ab) are associated with higher prevalence of multiple diabetes-related autoantibodies in adults with type 1 diabetes. Diabetes Res Clin Pract 146: 313-320.

4. Shi X, Huang G, Wang Y, Liu Z, Deng C, et al. (2019) Tetraspanin 7 autoantibodies predict progressive decline of beta cell function in individuals with LADA. Diabetologia 62: 399-407.

5. Husebye ES, Anderson MS, Kampe O (2018) Autoimmune polyendocrine syndromes. $N$ Engl J Med 378: 1132 1141.

6. Wang W, Mao J, Zhao J, Lu J, Yan L, et al. (2018) Decreased thyroid peroxidase antibody titer in response to selenium supplementation in autoimmune thyroiditis and the influence of a SEPP gene polymorphism: a prospective, multicenter study in China. Thyroid.

7. Yuan J, Zhou C, Gao J, Li J, Yu F, et al. (2017) Prevalence of celiac disease autoimmunity among adolescents and young adults in China. Clin Gastroenterol Hepatol 15: 1572-1579. e1.

8. Kaspers S, Kordonouri O, Schober E, Grabert M, Hauffa BP, et al. (2004) Anthropometry, metabolic control, and thyroid autoimmunity in type 1 diabetes with celiac disease: a multicenter survey. J Pediatr 145: 790-795.

9. Manso J, Pezzani R, Scarpa R, Gallo N, Betterle C (2018) The natural history of autoimmune Addison's disease with a non-classical presentation: a case report and review of literature. Clin Chem Lab Med 56: 896-900.

10. Mohn A, Di Michele S, Di Luzio R, Tumini S, Chiarelli F (2002) The effect of subclinical hypothyroidism on metabolic control in children and adolescents with type 1 diabetes mellitus. Diabet Med 19: 70-73.

11. Dost A, Rohrer TR, Frohlich-Reiterer E, Bollow E, Karges B, et al. (2015) Hyperthyroidism in 276 children and adolescents with type 1 diabetes from Germany and Austria. Horm Res Paediatr 84: 190-198. 
12. Frohlich-Reiterer EE, Hofer S, Kaspers S, Herbst A, Kordonouri O, et al. (2008) Screening frequency for celiac disease and autoimmune thyroiditis in children and adolescents with type 1 diabetes mellitus - data from a German/Austrian multicentre survey. Pediatr Diabetes 9: 546-553.

13. Winter WE, Schatz DA (2011) Autoimmune markers in diabetes. Clin Chem 57: 168-175.

14. Zhou Z, Xiang Y, Ji L, Jia W, Ning G, et al. (2013) Frequency, immunogenetics, and clinical characteristics of latent autoimmune diabetes in China (LADA China study): a nationwide, multicenter, clinic-based crosssectional study. Diabetes 62: 543-550.

15. Xiang Y, Huang G, Zhu Y, Zuo X, Liu X, et al. (2018) Identification of autoimmune type 1 diabetes and multiple organ-specific autoantibodies in adult-onset non-insulinrequiring diabetes in China: a population-based multicentre nationwide survey. Diabetes Obes Metab 21: 893-902.

16. Huang G, Xiang Y, Pan L, Li X, Luo S, et al. (2013) Zinc transporter 8 autoantibody (ZnT8A) could help differentiate latent autoimmune diabetes in adults (LADA) from phenotypic type 2 diabetes mellitus. Diabetes Metab Res Rev 29: 363-368.

17. Barker JM, Ide A, Hostetler C, Yu L, Miao D, et al. (2005) Endocrine and immunogenetic testing in individuals with type 1 diabetes and 21-hydroxylase autoantibodies: Addison's disease in a high-risk population. $J$ Clin Endocrinol Metab 90: 128-134.

18. Kochummen E, Marwa A, Umpaichitra V, Perez-Colon S, Chin VL (2018) Screening for autoimmune thyroiditis and celiac disease in minority children with type 1 diabetes. $J$ Pediatr Endocrinol Metab 31: 879-885.

19. Singh P, Seth A, Kumar P, Sajjan S (2017) Coexistence of celiac disease \& type 1 diabetes mellitus in children. Indian J Med Res 145: 28-32.

20. Chantzichristos D, Persson A, Eliasson B, Miftaraj M, Franzen S, et al. (2018) Incidence, prevalence and seasonal onset variation of Addison's disease among persons with type 1 diabetes mellitus: nationwide, matched cohort studies. Eur J Endocrinol 178: 113-120.

21. Glowinska-Olszewska B, Michalak J, Luczynski W, Del
Pilar Larosa M, Chen S, et al. (2016) Organ-specific autoimmunity in relation to clinical characteristics in children with long-lasting type 1 diabetes. $J$ Pediatr Endocrinol Metab 29: 647-656.

22. Yang L, Luo S, Huang G, Peng J, Li X, et al. (2010) The diagnostic value of zinc transporter 8 autoantibody (ZnT8A) for type 1 diabetes in Chinese. Diabetes Metab Res Rev 26: 579-584.

23. McLaughlin KA, Richardson CC, Ravishankar A, Brigatti C, Liberati D, et al. (2016) Identification of tetraspanin-7 as a target of autoantibodies in type 1 diabetes. Diabetes 65: 1690-1698.

24. Walther D, Eugster A, Jergens S, Gavrisan A, Weinzierl C, et al. (2016) Tetraspanin 7 autoantibodies in type 1 diabetes. Diabetologia 59: 1973-1976.

25. Luo S, Li X, Huang G, Xie Z, Xiang Y, et al. (2020) Distinct two different ages associated with clinical profiles of acute onset type 1 diabetes in Chinese patients. Diabetes Metab Res Rev 36: e3209.

26. Jonsdottir B, Larsson C, Lundgren M, Ramelius A, Jonsson I, et al. (2018) Childhood thyroid autoimmunity and relation to islet autoantibodies in children at risk for type 1 diabetes in the diabetes prediction in skane (DiPiS) study. Autoimmunity 51: 228-237.

27. Jonsdottir B, Andersson C, Carlsson A, Delli A, Forsander $\mathrm{G}$, et al. (2013) Thyroid autoimmunity in relation to islet autoantibodies and HLA-DQ genotype in newly diagnosed type 1 diabetes in children and adolescents. Diabetologia 56: $1735-1742$.

28. Zampetti S, Capizzi M, Spoletini M, Campagna G, Leto $\mathrm{G}$, et al. (2012) GADA titer-related risk for organ-specific autoimmunity in LADA subjects subdivided according to gender (NIRAD study 6). J Clin Endocrinol Metab 97: 3759-3765.

29. Jin P, Huang G, Lin J, Yang L, Xiang B, et al. (2011) High titre of antiglutamic acid decarboxylase autoantibody is a strong predictor of the development of thyroid autoimmunity in patients with type 1 diabetes and latent autoimmune diabetes in adults. Clin Endocrinol (Oxf) 74: 587592. 